\title{
Artículo
}

\section{Factores asociados a la productividad científica de docentes investigadores ${ }^{*}$}

Factors associated with the scientific productivity of researchers

Juan Jacobo Sarmiento Tovar ${ }^{* *}$

\section{Resumen}

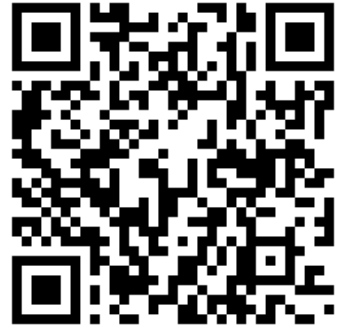

Los cambios estructurales en materia política en el Ecuador en los últimos 10 años, han provocado que las condiciones en la que opera la economía se encuentren en modificación constante, esto ha obligado a que, las Asociaciones Sociales replanteen un modelo productivo diferente a los esquemas tradicionales, para adaptarse a las nuevas tendencias de organización social. Frente a esta situación, el presente trabajo tiene como principal objetivo, Promover el desarrollo de una gestión moderna mediante un modelo de asociatividad para el sector pesquero artesanal de los puertos ubicados en la Comuna Chanduy, Santa Rosa y La Caleta en la Provincia de Santa Elena de la República del Ecuador. Unos de los factores que tiene mayor influencia en las Asociaciones es la globalización económica, lo obliga a las empresas a aplicar estrategias con el único fin de lograr una mayor eficiencia.. La metodología de investigación se caracteriza por utilizar un enfoque cuantitativo y cualitativo, mediante la aplicación de método deductivo y analítico, lo que permite medir las percepciones y expectativas de los Uno de los principales objetivos de las instituciones de educación superior (IES) es la producción de conocimiento científico, no solamente por ser un aporte fundamental al desarrollo y bienestar de la sociedad, sino también un importante indicador de calidad y, por ende, una ventaja competitiva dentro del

\footnotetext{
* Artículo original derivado del Proyecto "Educación universitaria en asociaciones" Financiado por la Universitaria Minuto de Dios entre mayo 2018 y mayo 2019

** Docente Universitario. Corporación Universitaria Minuto de Dios. Bogota, Colombia. E-mail: juan.sarmiento@uniminuto.edu. ORCID: http://orcid.org/0000-0003-0426-5981
} 
mercado educativo. Analizar los factores asociados con la actividad investigativa de los docentes de la institución, que fomentan o dificultan la productividad científica. La investigación tiene una metodología cuantitativa-descriptiva. Se analizaron ocho factores representados en cuarenta indicadores. Se determinan ocho factores que impactan la producción científica, ya sea fomentándola o dificultándola: Apoyo económico a la investigación, Asignación de tiempo, Competencias investigativas, Concentración investigativa, Desarrollo personal y profesional, Divulgación y socialización, Dotación tecnológica y Trabajo en equipo, redes y convenios.

Palabras clave: producción científica, productividad científica, investigación, factores

\begin{abstract}
One of the main objectives of higher education institutions (HEI) is the production of scientific knowledge, not only because it is a fundamental contribution to the development and welfare of society, but also an important indicator of quality and, therefore, an advantage Competitive within the educational market. Analyze the factors associated with the research activity of teachers of the institution, which encourage or hinder scientific productivity. The research has a quantitative-descriptive methodology. Eight factors represented in forty indicators were analyzed. Eight factors are determined that impact scientific production, the sea encouraging or hindering: Economic support for research, Time allocation, Research skills, Research concentration, Personal and professional development, Dissemination and socialization, Technological endowment and Teamwork, networks and agreements.
\end{abstract}

Key words: scientific production, scientific productivity, research, factors.

\title{
Introducción
}

La importancia creciente del conocimiento es uno de los rasgos fundamentales de la sociedad contemporánea, convirtiéndose en un tema predominante dentro de las actividades productivas y en una base fundamental para el fortalecimiento de sectores económicos en 
los cuales es común la inversión en investigación científica, dentro de estas dinámicas, tanto la información, como el conocimiento, pasan a convertirse en constituyentes del capital Krüger, (2006); Tünnerrnann y de Souza, (2003). A partir de esto, se concluye que la producción de conocimiento, particularmente científico, es un factor fundamental para el desarrollo económico y social de los países.

Teniendo en cuenta que las instituciones de educación superior generan la mayoría de la producción científica en el mundo Pereira, Casanova y Pire (2014), se puede entonces afirmar que éstas son entidades primordiales en los procesos de desarrollo anteriormente mencionados, lo que crea sobre ellas una gran presión en términos del cumplimiento de indicadores de producción científica.

En el contexto colombiano, las instituciones de educación superior deben responder a los lineamientos del Sistema Nacional de Acreditación, cuyo objetivo fundamental es garantizar la calidad de la educación (Ley 30 de diciembre 28 de 1992, Artículo 53). Sobre este principio se constituyen, el Registro Calificado y la Acreditación de Alta Calidad, los dos son mecanismos que le adjudican un alto valor a las condiciones que permiten la producción científica. (Ministerio de Educación, 2010; Consejo Nacional de Acreditación, 2014). De esta manera, la generación de productos científicos y académicos se ha convertido en una necesidad para las universidades, llevándolas a invertir importantes recursos y a tomar decisiones estratégicas para aumentar la capacidad de producción científica de su cuerpo profesoral.

Otro elemento fundamental que deben tener en cuenta las universidades colombianas, es la compleja estructura conceptual y normativa determinada por Colciencias (Departamento Administrativo de Ciencia, Tecnología e Innovación), la cual determina las características y tipologías de los productos científicos, así como los procedimientos y condiciones, para que éstos sean reconocidos Colciencias (2015). De tal manera que si la universidad y los mismos profesores quieren ver reflejadas sus esfuerzos académicos e investigativos en productos científicos, deben tener especial cuidado con los lineamientos mencionados. 
Bajo las dinámicas y condiciones anteriormente descritas, es importante para las universidades, que su planta docente tenga la capacidad de generar conocimiento científico de alta calidad y en cantidades suficientes, para lo cual es fundamental tener un entendimiento, lo más amplio posible, de los factores relacionados con el proceso de producción científica.

Existen también particularidades que respaldan la necesidad de generar dicho entendimiento en Corporación Universitaria Minuto de Dios - uniminuto, Centro Regional Zipaquirá (en adelante UNIMINUTO CRZ), puesto que se han observado fenómenos de importancia entre los años 2014 y 2017. En primer lugar, se ha evidenciado un aumento del número de horas asignadas para investigación y una leve disminución en la cantidad de proyectos de investigación, al mismo tiempo, la cantidad de productos por proyecto es muy baja, existiendo proyectos que no generaron ninguna publicación. Adicionalmente, se puede ver un fenómeno de concentración investigativa, término utilizado por Mendoza (2012), para identificar el estado en donde una minoría de investigadores generan el grueso de la producción científica.

A partir de la necesidad de entender las dinámicas y circunstancias involucradas en los procesos de producción científica de la institución, el objetivo del presente estudio es analizar los factores asociados con la actividad investigativa de los docentes en UNIMINUTO CRZ, que fomentan o dificultan la productividad científica.

Respecto a este tema, se encuentra una amplia variedad de investigaciones en la literatura científica reciente, que intentan dar cuenta del fenómeno de la producción científica en universidades y grupos de investigación. Dentro de este grupo de investigaciones se encuentran algunas especialmente orientadas a determinar las relaciones existentes entre diversos factores y la productividad científica. Tal es el caso de Rueda-Barrios y Rodenes-Adam (2016), cuyo trabajo, de alcance cuantitativo-explicativo, se orientó al estudio de la relación entre la producción científica de los grupos de investigación adscritos a universidades colombianas y tres variables que consideraron determinantes: cultura organizacional, gestión del conocimiento y capital tecnológico, en este caso se buscó comprobar 
la relación causa-efecto de estos tres factores sobre la producción científica. Los resultados muestran que efectivamente existen indicadores pertenecientes a dichos factores que explican la producción científica de los investigadores.

Con el mismo alcance del estudio anteriormente descrito, una investigación de Robles-Jopia, Sánchez-Ortiz y Ramírez-Correa (2016) se orienta a construir un modelo que relacione factores institucionales y personales, con la producción científica de los investigadores, al igual que en el caso anterior, se validaron relaciones causa-efecto entre ésta y algunos indicadores pertenecientes a los factores mencionados. Da la misma manera, Parra, Monge y Vildósola (2009), realizan una investigación orientada a identificar los factores personales, laborales y académicos que se relacionan con la producción científica, en este caso, de los médicos gastroenterólogos que desempeñan sus labores profesionales en Lima, Perú. El estudio identificó que el tipo de búsqueda bibliográfica, la facilidad para la investigación en el trabajo, el grado de comprensión de artículo científico, el pertenecer a una sociedad científica y el número de empleos, son factores asociados a la producción científica de los investigadores.

En una dirección parecida, Escobar-Pérez, García-Meca y LarránJorge (2014) se proponen descubrir el origen de la diferencia entre la producción científica de los profesores pertenecientes a la carrera de Contabilidad y la producción en otras carreras. Dicho estudio les permitió identificar un conjunto de factores que impactaban negativamente la productividad, dándole explicación a las diferencias planteadas. Con el mismo alcance correlacional, pero en una dirección distinta, Acevedo, Montero y Duran (2016), realizan un estudio en el que se busca establecer la relación entre la percepción de calidad en la enseñanza que tienen los alumnos respecto a sus profesores en una universidad colombiana y la productividad académica de éstos últimos. El estudio identifico una relación positiva entre estos dos factores, pero condicionada por la carrera universitaria a la que pertenece el alumno. Los trabajos mencionados anteriormente, logran determinar importantes relaciones entre la producción científica y diversas variables identificadas, permitiendo establecer, en la mayoría de los casos, 
relaciones causa-efecto que dan cuanta de un entendimiento profundo de algunas dinámicas que se presentan en los procesos de generación de conocimiento científico, sin embargo es amplio y complejo el sistema de elementos que están relacionados con la producción de conocimiento y en muchos casos, es conveniente generar enfoques exploratorios y descriptivos que permitan conocer en un sentido más amplio el fenómeno, abriéndose a la posibilidad de encontrar en el camino nuevas variables relacionadas con el fenómeno estudiado, a continuación se presentan importantes trabajos orientados en esta dirección.

Desde un enfoque cuantitativo y descriptivo, Sánchez Hernández, de la Torre, Sorzano, Ramos y Durán (2016), realizan un estudio que busca caracterizar factores que puedan limitar la producción científica. Al igual que Parra, Monge y Vildósola (2009), este trabajo se enfoca en profesionales de la salud. Las categorías estudiadas son: intereses de los investigadores, sus necesidades profesionales, el proceso de organización y la gestión del proceso de publicación científica. Los resultados permiten identificar factores limitantes importantes como lo son las limitaciones de tiempo y los requisitos exigidos por las editoriales a la hora de someter sus escritos.

Por su parte Mendoza (2012), realiza un estudio con enfoque cualitativo, orientado a profesores de la facultad de medicina de una universidad UNMSM, en Lima, Perú. En éste, busca la identificación de los factores que puedan estar conexos con la producción de publicaciones científicas. Cabe destacar que sus resultados develan factores facilitadores y bloqueadores, junto con circuitos de relaciones que refuerzan dichos factores. Al igual que la investigación anteriormente mencionada, Sogi, Perales, Anderson y Bravo (2002), realiza una investigación orientada a identificar factores relacionados con la producción científica en docentes investigadores de la facultad de medicina de una universidad UNMSM. En este caso, el enfoque metodológico es mixto y establece las categorías personales, institucionales y contextuales, dentro de las cuales identificaron diversos factores limitantes.

En otra investigación de enfoque mixto, Narváez y Burgos (2011) se enfocan la productividad investigativa de los docentes, buscando establecer los factores que la condicionan, sus resultados muestran 
que los intereses particulares juegan un papel fundamental, por otro lado, identifican que las actividades administrativas y aquellas relacionadas con la docencia predominan sobre las de carácter investigativo, por último concluyen que la ausencia de políticas académicas que apoyen apropiadamente los procesos investigativos, desestimula la investigación. Utilizando el mismo enfoque mixto, Flores, Ordóñez, Viramontes (2015), presentan un estudio orientado a identificar los factores que se relacionan con la producción científica en las instituciones de educación superior, ya sea inhibiéndola o alentándola, en este sentido, se asemeja a los objetivos de investigaciones como las de Mendoza (2012). La población específica de esta investigación, la comprenden los profesores de tiempo completo de las áreas económicoadministrativas.

Desde una perspectiva distinta, un estudio de Gómez-Vargas y García (2015), se enfoca en determinar factores facilitadores e inhibidores asociados a la gestión del conocimiento practicado por los grupos de investigación pertenecientes a una universidad colombiana. Respecto al manejo del concepto gestión del conocimiento, la investigación se asemeja a la de Rueda-Barrios y Rodenes-Adam (2016), generando un aporte importante, en la medida en que en ésta última se comprueba la estrecha relación entre la capacidad de producción científica y las buenas prácticas en gestión del conocimiento. Esta investigación permite comprobar factores teóricos encontrados en la literatura y también identificar nuevos factores como lo son el tiempo dedicado a la investigación, así como la cantidad de personas que se dedican a ella. Tanto en este estudio como en el de Flores, Ordóñez y Viramontes (2015) y Mendoza (2012), se evidencia la importancia de identificar factores que actúen de manera positiva y negativa frente a la producción científica, esto permite la toma de decisiones estratégicas en términos administrativos y académicos que estimulen la cantidad y calidad de las publicaciones al interior de las universidades. 


\section{Materiales y métodos}

Se determina el uso de un enfoque cuantitativo para el abordaje del presente estudio. Como primera medida, se recogen la totalidad de factores teóricos encontrados en los antecedentes, de los cuales se seleccionan 8 que resultan pertinentes respecto a la realidad de la población específicamente estudiada. Posteriormente, se miden los factores teóricos seleccionados en la población de profesores a estudiar, la cual tiene un tamaño de 162 individuos. La recolección de datos se realiza por medio de una encuesta vía formulario de Google que consta de 54 puntos, los primeros 14 son de caracterización de la población en términos demográficos, laborales $\mathrm{y}$ profesionales, los siguientes 40 puntos hacen referencia a los indicadores que describen los 8 factores seleccionados en la fase anterior como se observa en la Tabla 1, para su medición se utiliza una escala Likert con 5 grados de respuesta. La encuesta fue realizada vía formulario electrónico a la totalidad de la población, de la cual se obtuvo respuesta de 47 profesores, se entiende entonces esta muestra como no probabilística. A partir de las encuestas recibidas, se analizaron los datos de manera descriptiva por medio de tablas de frecuencia mediante el programa SPSS.

En cuanto a la validación del instrumento, se verificó la validez mediante juicio de expertos, en el que participaron tres investigadores experimentados, el instrumento seleccionado es aportado por Corral (2009). Para verificar la confiabilidad, se realizó una prueba piloto con 33 profesores, la cual arrojó un Alfa de Cronbach de 0,84.

\section{Resultados}

Como se explicó anteriormente, el estudio cuantitativo consta de una encuesta de 54 puntos, de los cuales 14 son para la caracterización de la población y 40 constituidos por los indicadores de los 8 factores identificados en la literatura. En cuanto a la medición de las variables que corresponden a los indicadores de los factores seleccionados, sus resultados se pueden observar en la Tabla 1. 
Tabla 1

Indicadores escala de Likert

\begin{tabular}{|c|c|c|c|c|c|c|}
\hline & Indicador & 1 & 2 & 3 & 4 & 5 \\
\hline F1 V15 & $\begin{array}{l}\text { Formación en programas de } \\
\text { posgrado }\end{array}$ & $4 \%$ & $9 \%$ & $15 \%$ & $43 \%$ & $30 \%$ \\
\hline V16 & Asistencia a eventos científicos & $2 \%$ & $13 \%$ & $34 \%$ & $38 \%$ & $13 \%$ \\
\hline V17 & $\begin{array}{l}\text { Financiación de proyectos y } \\
\text { semilleros }\end{array}$ & $4 \%$ & $9 \%$ & $45 \%$ & $34 \%$ & $9 \%$ \\
\hline V18 & Apoyo producción científica & $2 \%$ & $13 \%$ & $38 \%$ & $32 \%$ & $15 \%$ \\
\hline V19 & Formación en nuevas tecnologías & $2 \%$ & $4 \%$ & $21 \%$ & $53 \%$ & $19 \%$ \\
\hline F2 V20 & $\begin{array}{l}\text { Gestión procesos académicos (p.e. } \\
\text { Autoevaluación y acreditación) }\end{array}$ & $2 \%$ & $13 \%$ & $17 \%$ & $43 \%$ & $26 \%$ \\
\hline V21 & $\begin{array}{l}\text { Carga académica (Clases, currículo } \\
\text { y diseño pedagógico) }\end{array}$ & $26 \%$ & $36 \%$ & $15 \%$ & $21 \%$ & $2 \%$ \\
\hline $\mathrm{V} 22$ & Horas asignadas para investigación & $4 \%$ & $6 \%$ & $32 \%$ & $38 \%$ & $19 \%$ \\
\hline V23 & Posgrados & $13 \%$ & $34 \%$ & $34 \%$ & $15 \%$ & $4 \%$ \\
\hline V24 & Transferencia de tiempo & $11 \%$ & $40 \%$ & $34 \%$ & $11 \%$ & $4 \%$ \\
\hline F3 V25 & $\begin{array}{l}\text { Uso de herramientas de búsqueda } \\
\text { de información }\end{array}$ & $2 \%$ & $26 \%$ & $11 \%$ & $36 \%$ & $26 \%$ \\
\hline V26 & Conocimientos metodológicos & $2 \%$ & $26 \%$ & $15 \%$ & $43 \%$ & $15 \%$ \\
\hline V27 & Productos Colciencias & $9 \%$ & $23 \%$ & $23 \%$ & $34 \%$ & $11 \%$ \\
\hline V28 & Revistas indexadas & $11 \%$ & $26 \%$ & $17 \%$ & $36 \%$ & $11 \%$ \\
\hline
\end{tabular}




\begin{tabular}{|c|c|c|c|c|c|c|}
\hline V29 & Escritura científica y argumentativa & $2 \%$ & $15 \%$ & $30 \%$ & $43 \%$ & $11 \%$ \\
\hline V30 & Inglés & $15 \%$ & $34 \%$ & $13 \%$ & $23 \%$ & $15 \%$ \\
\hline V31 & Software & $4 \%$ & $28 \%$ & $19 \%$ & $36 \%$ & $13 \%$ \\
\hline V32 & Aplicativo Cvlac & $6 \%$ & $15 \%$ & $17 \%$ & $40 \%$ & $21 \%$ \\
\hline F4 V33 & $\begin{array}{l}\text { Oportunidades } \\
\text { investigaciones }\end{array}$ & r $15 \%$ & $32 \%$ & $36 \%$ & $17 \%$ & $0 \%$ \\
\hline V34 & Oportunidades publicar resultados & $9 \%$ & $32 \%$ & $38 \%$ & $21 \%$ & $0 \%$ \\
\hline V35 & $\begin{array}{l}\text { Capacidades y conocimientos } \\
\text { investigativos }\end{array}$ & $11 \%$ & $36 \%$ & $32 \%$ & $21 \%$ & $0 \%$ \\
\hline F5 V36 & 5 Desarrollo académico & $0 \%$ & $2 \%$ & $17 \%$ & $28 \%$ & $53 \%$ \\
\hline V37 & Ingresos & $0 \%$ & $11 \%$ & $19 \%$ & $38 \%$ & $32 \%$ \\
\hline $\mathrm{V} 38$ & Prestigio y reconocimiento & $0 \%$ & $2 \%$ & $6 \%$ & $38 \%$ & $53 \%$ \\
\hline V39 & $\begin{array}{l}\text { Motivación } \\
\text { investigativas }\end{array}$ & $2 \%$ & $15 \%$ & $26 \%$ & $28 \%$ & $30 \%$ \\
\hline V40 & Motivación - generar productos & $2 \%$ & $21 \%$ & $30 \%$ & $19 \%$ & $28 \%$ \\
\hline F6 V41 & Reconocimientos & $2 \%$ & $11 \%$ & $45 \%$ & $30 \%$ & $13 \%$ \\
\hline V42 & $\begin{array}{l}\text { Organización } \quad \text { de } \\
\text { científicos }\end{array}$ & $2 \%$ & $4 \%$ & $43 \%$ & $43 \%$ & $9 \%$ \\
\hline V43 & Resultados & $2 \%$ & $17 \%$ & $26 \%$ & $43 \%$ & $13 \%$ \\
\hline V44 & Propuestas de investigación & $4 \%$ & $4 \%$ & $21 \%$ & $47 \%$ & $23 \%$ \\
\hline V45 & Espacios de publicación & $6 \%$ & $21 \%$ & $21 \%$ & $40 \%$ & $11 \%$ \\
\hline
\end{tabular}




\begin{tabular}{|c|c|c|c|c|c|c|}
\hline F7 V46 & Equipos y software & $11 \%$ & $23 \%$ & $45 \%$ & $17 \%$ & $4 \%$ \\
\hline V47 & Laboratorios & $17 \%$ & $26 \%$ & $45 \%$ & $9 \%$ & $4 \%$ \\
\hline V48 & Bases de datos & $4 \%$ & $4 \%$ & $47 \%$ & $36 \%$ & $9 \%$ \\
\hline V49 & Espacios físicos & $26 \%$ & $49 \%$ & $17 \%$ & $6 \%$ & $2 \%$ \\
\hline F8 V50 & $\begin{array}{l}\text { Disposición a } \\
\text { conocimientos }\end{array}$ & $r_{4 \%}$ & $19 \%$ & $38 \%$ & $30 \%$ & $9 \%$ \\
\hline V51 & $\begin{array}{l}\text { Se promueve } \\
\text { multidisciplinar }\end{array}$ & $0 \%$ & $21 \%$ & $32 \%$ & $38 \%$ & $9 \%$ \\
\hline V52 & $\begin{array}{l}\text { Se promueve la creación grupos de } \\
\text { investigación }\end{array}$ & $2 \%$ & $11 \%$ & $26 \%$ & $53 \%$ & $9 \%$ \\
\hline V53 & $\begin{array}{l}\text { Se promueven } \\
\text { interinstitucionales }\end{array}$ & $4 \%$ & $11 \%$ & $43 \%$ & $36 \%$ & $6 \%$ \\
\hline V54 & $\begin{array}{l}\text { Se promueve participación en redes } \\
\text { científicas }\end{array}$ & $0 \%$ & $13 \%$ & $51 \%$ & $28 \%$ & $9 \%$ \\
\hline
\end{tabular}

Elaboración propia

Factor 1: Apoyo económico a la investigación

En cuanto a el primer factor (variables 15 a 19), este recibe un puntaje positivo del $57 \%$, al tomar el promedio de sus indicadores en las opciones 4 y 5. Si se tiene en cuenta que, Flores, Ordóñez, Viramontes (2015), lo identifican como uno de los principales factores inhibidores en su investigación, los resultados son bastante satisfactorios respecto a este tema. Para este factor, los indicadores de Formación en programas de posgrado y Formación en nuevas tecnologías obtienen los puntajes más altos con $73 \%$ y $72 \%$ respectivamente, tomando las opciones 4 y 5 en conjunto. Al respecto, Narváez y Burgos (2011), miden los factores Escasa formación y Falta de adiestramiento, en los que obtuvieron ambos un puntaje de $27 \%$, lo que significa que el $73 \%$ no cree que estos 
factores actúen como condiciones negativas, este resultado presenta una alta coincidencia con el presente estudio.

Los datos muestran que en promedio $37 \%$ de los encuestados le asignaron valores entre 4 y 5 , al igual que la suma de los puntajes 1 y 2. Según estos resultados, no existe una percepción con una tendencia clara respecto al tema de la asignación de tiempos. Analizando las variables separadamente, se observa que la Carga gestión académica y las Horas de investigación tienen un puntaje positivo con $68 \%$ y $58 \%$ respectivamente en sus opciones 4 y 5 . Al respecto, Flores, Ordóñez, Viramontes (2015), identifica el exceso de carga académica administrativa y de gestión como un factor que inhibe la investigación científica, se puede ver entonces una aparente discrepancia en los resultados, sin embargo se debe tener en cuenta que la variable 21, Carga académica, tiene una calificación evidentemente negativa al sumar el $62 \%$ en sus puntajes 1 y 2 . La diferencia radica en que el estudio de Flores, Ordóñez, Viramontes (2015) no diferencia las cargas académicas de las administrativas. Por último, se debe notar que los indicadores Posgrados $\mathrm{y}$ Transferencia de tiempo, son también negativos al sumar 47\% y 51\% en sus puntajes 1 y 2 .

El tercer factor, está compuesto por ocho variables (variables 25 a 32). En este caso también se observa en general una percepción positiva por parte de los encuestados. Es de resaltar el comportamiento de la variable 30 que muestra un peso de $48.9 \%$ en los puntajes 1 y 2 frente a un $38.3 \%$ en los puntajes 4 y 5 . Lo que significa que los profesores tienden a percibir que sus capacidades en el idioma inglés son insuficientes para sus labores investigativas, sin embargo, la tendencia no es muy marcada. Al respecto de este tema, Gómez-Vargas y García (2015), evalúan dos factores personales relacionados: Competencias y Métodos y herramientas. Los resultados apuntan a que estos factores en general tienen un impacto positivo en la gestión del conocimiento, lo que genera una coincidencia respecto a la percepción de los profesores encuestados.

El cuarto factor está compuesto por las variables 33, 34 y 35 . Al contrario del anterior, éste muestra una fuerte tendencia negativa. Cabe resaltar también el hecho de que se generó un puntaje alto en la opción "neutral", es decir, aquellas que tuvieron un puntaje de 3 dentro de la escala. Se puede ver que en general el 35.5\% de los 
encuestados dio una respuesta neutral para las variables de este factor. Esto puede responder al hecho de que se requiere una cierta experiencia en los procesos de investigación en la institución para tener una postura clara respecto al tema de la concentración. Respecto a este tema, Mendoza (2012) identifica el factor Concentración como reforzador de los factores bloqueadores de la producción científica, por lo tanto, coincide con los resultados del presente estudio.

Este factor está compuesto por las variables 36 a 40 y se orienta a explorar el valor percibido por los profesores respecto a la producción científica y su impacto en diversas esferas de su vida personal y profesional. El análisis de los datos permite determinar que los profesores le dan un alto valor a la investigación desde las esferas personales y profesionales mencionadas. Se evidencia que el $70 \%$ del peso de la muestra se encuentran entre los puntajes 4 y 5 de la escala. Es importante notar que, si bien todas las variables tienen una claro balance positivo, la variable 40 presenta el mayor índice negativo, es decir un $24 \%$ entre los puntajes 1 y 2 , adicionalmente presenta el porcentaje más alto de neutralidad (30\%), esto lleva a pensar que no obstante los profesores le asignan un valor positivo a la investigación, la generación de productos científicos no genera una certeza de forma tan contundente, probablemente por las dificultades observadas en la práctica por la Coordinación de Investigación, en donde los investigadores no muestran mucha claridad y fluidez al momento de traducir sus actividades investigativas a productos científicos. Respecto a este tema, RuedaBarrios y Rodenes-Adam (2016), evalúan la variable Crecimiento profesional y personal, que hace parte de la cultura motivadora, para la cual comprueban una alta relación positiva con la producción científica, esto nos habla de un alto potencial respecto a este factor como factor que promueve la producción científica en Uniminuto CRZ.

El sexto factor, lo conforman las variables de la 41 a la 45 . Su objetivo es medir la percepción de los profesores respecto a la labor de la institución frente a estrategias de divulgación necesarias para el adecuado desarrollo de la cultura y procesos investigativos. En este caso se observa una percepción bastante positiva frente a este factor con un $54 \%$ de peso entre los puntajes 4 y 5 de la escala. Un 
aspecto importante es la fuerte neutralidad que se observa frente a las afirmaciones de las variables 41 y 42 , con un $45 \%$ y $43 \%$ respectivamente. Esto podría significar un desconocimiento que señala la existencia de un fenómeno de exclusión o "población periférica" de profesores que no están integrados en una red de comunicación para la divulgación y socialización de algunos temas. Es importante entonces ahondar más en estos factores en el futuro para tener una mejor comprensión de dicha neutralidad. Por último, es de resaltar el comportamiento positivo de la variable 44 que está relacionada con la socialización de las convocatorias para proyectos y publicaciones, ya que este proceso comunicativo es clave para el cumplimiento de los indicadores de gestión de la Coordinación de Investigación. Rueda-Barrios y Rodenes-Adam (2016), manejan el factor Procesos de comunicación, dentro de la cultura participativa, que como ya se mencionó está relacionada de manera positiva con la producción científica. Adicionalmente, Sánchez Hernández, de la Torre, Sorzano, Ramos y Durán (2016) identifican el factor, Dar a conocer los resultados a la comunidad científica, como un facilitador de la producción científica.

El séptimo factor, está conformado por cuatro variables, de la 46 a la 49. Este factor tiene, en general, una percepción negativa con un $40 \%$ del peso en los puntajes 1 y 2 y sólo un $22 \%$ entre los puntajes 4 y 5 . Se destaca un alto índice de neutralidad con un promedio de $38 \%$ en el puntaje 3. Este comportamiento hace necesario un análisis más pormenorizado de las variables involucradas. Las variables 46 y 47, que apuntan a los temas de software, equipos y laboratorios, tienen un comportamiento muy parecido, en el que se evidencia una percepción negativa y al mismo tiempo un alto índice de neutralidad (45\%). La variable 48, tiene la particularidad de ser atípica respecto al comportamiento de las demás variables del factor. Esta variable tiene un comportamiento positivo con un $45 \%$ entre los puntajes $4 \mathrm{y}$ 5 frente a un $8.6 \%$ entre los puntajes 1 y 2 . Finalmente se tiene la variable 49 que sigue la tendencia negativa del grupo, pero de manera más contundente dado que tiene un peso del $75 \%$ entre los puntajes 1 y 2 de la escala, mientras que el $8.5 \%$ está entre los puntajes 4 y 5. Al respecto, Narváez y Burgos (2011), manejan el factor, Escaso apoyo tecnológico, como un posible condicionante que funcionara en contra de la producción científica. Cabe anotar que Rueda-Barrios y Rodenes-Adam (2016), comprueban que el capital 
tecnológico influye de manera positiva y significativa sobre la producción científica.

\section{Discusión}

Los datos obtenidos en la presente investigación, al igual que los resultados de los diversos estudios identificados en la literatura han permitido dilucidar diversos factores y el impacto favorable o desfavorable que tienen sobre la producción científica en UNIMINUTO CRZ. Sin embargo, es importante notar que existen una serie de indicadores con puntajes altos de neutralidad, lo que puede indicar desconocimiento del tema tratado. Igualmente existen factores cuyos indicadores ofrecen resultados discordantes. Es importante en futuras investigaciones profundizar en estos fenómenos para entender el origen de estos resultados.

Si bien el alcance descriptivo ha entregado una cantidad significativa de información, es importante buscar el entendimiento de la forma en que las variables estudiadas se relacionan con la producción científica de manera cuantitativa, es decir con la cantidad de productos científicos que los investigadores estudiados generan. Con esto se podrá llegar a un entendimiento explicativo del fenómeno de la productividad científica de los investigadores.

Uno de los factores que de manera más clara está produciendo un efecto negativo en la producción científica es el de Concentración (factor 4), éste es definido por Mendoza (2012) como un fenómeno que "se da por el porcentaje de docentes que explican la producción de una Facultad" (p.141). Es importante ahondar en sus efectos tanto productivos como psicológicos, ya que quedan indicios de que puede ser un causante de aislamiento y segregación y puede generar un efecto de "profecía autocumplida" que impulsa cada vez más a los investigadores sobresalientes y discrimina a aquellos que no han logrado desarrollar las competencias y la experiencia necesaria en términos investigativos.

Debido a los resultados y a la naturaleza del fenómeno, se hace necesario generar un modelo explicativo que tenga en cuenta las teorías del aprendizaje individual y organizacional, así como las teorías relacionadas con la gestión del conocimiento ya que se evidencia su pertinencia respecto a las dinámicas y factores estudiados. Un estudio que nos da pie para esta afirmación es el de 
Gómez-Vargas y García (2015), titulado "Factores influyentes de la gestión del conocimiento en el contexto de la investigación universitaria" en el que los factores facilitadores e inhibidores identificados resultan muy adecuados para la comprensión del fenómeno.

\section{Conclusiones}

El apoyo económico a la investigación es un factor que fomenta la producción científica en la institución, acorde a la percepción de los profesores. Esta conclusión está sustentada por los resultados de los cinco indicadores que constituyen dicho factor, principalmente, Formación en programas de posgrado y Formación en nuevas tecnologías.

Asignación de tiempo, es un factor que no presenta congruencia entre los resultados descriptivos de sus indicadores. De esta manera se constituye como un factor que fomenta la investigación si se toman en cuenta los indicadores de Carga gestión académica y Horas de investigación, sin embargo, dificulta la producción científica si se toma en cuenta la Carga académica, la Asignación de tiempo para posgrados y la Transferencia de tiempo.

El factor competencias investigativas fomenta la producción científica. Los datos reflejan que los indicadores Uso de herramientas de búsqueda de información; Conocimientos metodológicos, Productos Colciencias, Revistas indexadas, Escritura científica y argumentativa; Software y Aplicativo Cvlac son identificados como variables que apoyan la producción científica. El único indicador con puntaje negativo fue Inglés, debido a que lo identifican como un falencia dentro de las competencias de los investigadores que dificulta la producción.

La concentración investigativa es un factor que dificulta la producción al restringir el acceso a oportunidades para generar investigaciones, publicar resultados y desarrollar Capacidades y conocimientos investigativos. Estos tres indicadores obtuvieron puntuaciones altas en las opciones 1 y 2 de la escala de Likert. 
En cuanto al factor que evalúa la percepción de desarrollo personal y profesional asociada a la producción científica, los resultados muestran que éste fomenta la producción científica. La totalidad de indicadores respaldan esta conclusión al tener puntajes altos en las opciones 4 y 5 de la escala: Desarrollo académico, Ingresos, Prestigio y reconocimiento, Motivación frente a las actividades investigativas y Motivación frente a la generación de productos científicos.

La divulgación y socialización de los resultados investigativos es percibida de forma positiva por los investigadores, de tal manera que este es un factor que fomenta la producción científica. Los cinco indicadores que la componen tienes puntuaciones positivas: Reconocimientos, Organización de Eventos científicos, Resultados, Propuestas de investigación y Espacios de publicación. Sin embargo, se debe tener en cuenta que las dos primeras tienen puntajes altos de neutralidad, lo que puede significar que existe un desconocimiento del tema por parte de los profesores de la institución.

La dotación tecnológica es un factor que no ofrece un resultado contundente respecto a los datos obtenidos. Para los indicadores de Equipos y software y Laboratorios, los puntajes de neutralidad son altos, mostrando también fuerza en las opciones 1 y 2 de la escala, lo que los identifica como variables que dificultan la producción. Bases de datos, es un indicador que también presenta un puntaje alto de neutralidad, pero también es fuerte en las opciones 4 y 5 de la escala, así que se puede afirmar que es una variable que fomenta la producción. Finalmente, el indicador Espacios, es el único que muestra con claridad un puntaje alto en las opciones 1 y 2 de la escala y baja neutralidad, lo que lo define claramente como una variable que dificulta la producción científica.

El trabajo en equipo, redes y convenios se identifica como un factor que fomenta la producción científica de los investigadores debido a que se comparten conocimientos, hay equipos multidisciplinares, nuevos grupos de investigación, convenios interinstitucionales y participación en redes científicas. 


\section{Referencias}

Acevedo, D., Montero, P., \& Duran, M. (2016). Análisis de la Productividad Académica de Profesores del Área de Ingeniería. Formación Universitaria, 9(2), 89-96.

Colciencias, Dirección de Fomento a la Investigación. (2015). Modelo de Medición de Grupos de Investigación, Desarrollo Tecnológico o de Innovación y de Reconocimiento de Investigadores del Sistema Nacional de Ciencia, Tecnología e Innovación. Obtenido de www.colciencias.gov.co:

\section{http://www.colciencias.gov.co/sites/default/files/upload/noticias/medicio} ndegrupos-actene2015.pdf

Consejo Nacional de Acreditación. (2014). Lineamientos para la Acreditación Institucional. Obtenido de www.cna.gov.co: https://www.cna.gov.co/1741/articles-186359_Lin_Ins_2014.pdf

Consejo Nacional de Acreditación. Consejo Nacional de Acreditación. (20 de mayo de 2019). Obtenido de https://www.cna.gov.co/1741/article186382.html

Corral, Y. (2009). Validez y confiabilidad de los instrumentos de investigación para la recolección del datos. Ciencias de la Educación, 19(33), 228-247.

Escobar-Pérez, B., García-Meca, E., \& Larrán-Jorge, M. (2014). Factores que inflluyen sobre la producción científica en Contabilidad en España: la opinión de los profesores universitarios de Contabilidad (II parte). Revista de Documentoación Científica, $37(2)$. doi:http://dx.doi.org/10.3989/redc.2014.2.1087

Fernández, L. (octubre de 2006). ¿Cómo analizar datos cualitativos? (U. d. Barcelona, Ed.) Butlletí LaRecerca. Obtenido de http://www.ub.edu/ice/recerca/fitxes/fitxa7-cast.htm

Flores, C., Ordoñez, A., \& Viramontes, O. (7, 8 y 9 de octubre de 2015). Factores que afectan la investigación científica en las instituciones de educación superior (área econímico-administrativa). México D.F., México.

Gómez-Vargas, M. Y García, M. (2015). Factores influyentes de la gestión del conocimiento en el contexto de la investigación universitaria. Información, cultura y sociedad, 33 (diciembre 2015), 29-46.

Krüger, K. (25 de septiembre de 2006). El concepto de la "Sociedad del Conocimiento". (U. d. Barcelona, Ed.) Revista Bibliográfica de Geografía 
y Ciencias Sociales, XI(683). Obtenido de http://www.ub.es/geocrit/b3w683.htm>. [ISSN 1138-9796]

Ley 30 de Diciembre 28 De 1992. Por el cual se organiza el servicio público de la Educación Superior. Congreso de Colombia.

Mendoza, P. (2012). Factores asociados a la producción de publicaciones por profesores de la Facultad de Medicina de la UNMSM. Acta Med Per, 29(3).

Ministerio de Educación Nacional. (20 de abril de 2010). Decreto 1295. Colombia. Obtenido de https://www.mineducacion.gov.co/1621/articles229430 archivo pdf decreto1295.pdf

NarvÁEZ, J., \& Burgos, J. (enero-abril de 2011). La productividad investigativa del docente universitario. Orbis. Revista científica de Ciencias Humanas, 6(18), 116-140. Obtenido de http://www.redalyc.org/articulo.oa?id=70918499006

Parra, V., Monge, E., \& Vildósola, H. (2009). Factores relacionados con la Producción Científica de los Médicos Gastroenterólogos en Lima - Perú. Gastroenterol, 29(3), 226-233.

Pereira, A., Casanova, M. Y Pire, R. (2014). Estudio comparativo de la producción científica de las universidades públicas y privadas venezolanas. Compendium, 17(32), 55-77.

Robles-Jopia, P., Sánchez-Ortiz, A., \& Ramírez-Correa, P. (enero-junio de 2016). Factores que influyen en la producción científica en la Universidad Católica del Norte. Universitas Gestão e TI, 6(1), 33-39. doi:10.5102/un.gti.v6il.4108

Rueda-Barrios, G. Y Rodenes-Adam, M. (2016). Factores determinantes en la producción científica de los grupos de investigación en Colombia. Revista Española de Documentación Científica, 39(1): e118. doi: http://dx.doi.org/10.3989/redc.2016.1.1198

Sánchez, E., De La Torre, G., Sorzano, G., Ramos, M., \& Durán, S. (2016). Factores limitantes de la producción científica en profesionales de la salud. MEDISAN, 20(1).

Sogi, C., Perales, A., Anderson, A., Y Bravo, E. (2002). El proceso de producción científica de los investigadores de la Facultad de Medicina, UNMSM. Anales de la Facultad de Medicina, 63(2), 115-124. [ISSN 1025 $-5583]$.

Tünnermann, C. Y De Souza, M. (2003). Desafíos de la Universidad en la Sociedad del Conocimiento, Cinco Años Después de la Conferencia 
Mundial sobre Educación Superior. Comité Científico Regional para America Latina y el Caribe del Foro de la UNESCO. UNESCO Forum Occasional Paper Series Paper no. 4 / S, Paris. 